\title{
Localization of Mobile Submerged Sensors using Lambert-W Function and Cayley-Menger Determinant
}

\author{
Anirban Paul ${ }^{1}$, Miad Islam², Md. Ferdousur Rahman ${ }^{3}$, Anisur Rahman ${ }^{4}$ \\ Department of Computer Science and Engineering \\ East West University, Dhaka \\ Bangladesh
}

\begin{abstract}
This paper demonstrates a new mechanism to localize mobile submerged sensors using only a single beacon node. In range-based localization, fast and accurate distance measurement is vital in underwater wireless sensor networks (UWSN). The knowledge of exact coordinates of the sensors is as important as the actuated data in underwater wireless sensor networks. Mostly bouncing technique is used to determine the distance between the beacon and the sensors. Moreover, to determine the coordinates, trilateration and multilateration technique is used; where using multiple beacons (usually three or more) is the most common approach. Nevertheless, because of many factors, this method gives less accurate results in distance measurements, which finally leads to determine erroneous coordinates. As TDOA is very ponderous to achieve in underwater environment because of time synchronization; again, using AOA is extremely difficult and challenging; TOA is the most common approach and is widely employed. However, it still needs precise synchronization. So, to determine the distances between beacon and sensor nodes, we have used a method based on Lambert-W function in this study, which is an approach based on RSS, and it avoids any synchronization. Besides, coordinates of the mobile sensors are calculated using CayleyMenger determinant. In this paper, the method is derived and the accuracy is verified by simulation results.
\end{abstract}

Keywords-Lambert-W function; Cayley-Menger determinant; submerged mobile sensor; single beacon; localization

\section{INTRODUCTION}

The underwater wireless communication is still a very challenging term in wireless communications. As radio signals cannot propagate much underwater, acoustic signals are widely used as a substitute. The need of knowing and observing the marine life is increasing rapidly. The data lays underwater could be of great use with precise Information about the location. Moreover, collecting those data is equally important for underwater surveillance, deep-sea exploration etc. Therefore, it is very important to collect those data using submerged sensors. In addition, according to [1], the accurate localization of sensors is vital for proper interpretation of the actuated data. In terrestrial condition, localization of wireless sensors has developed greatly and many mechanisms have been proposed. There are two categories: range-free and range-based schemes. Range-based scheme can give more accurate result than range-free scheme and most of the sensors nowadays have those characteristics. In this paper, we have studied on accuracy of submerged moving sensors coordinate, which has a wide range of application in practical, like pollutant tracking and estuary monitoring [2]. Moreover, as seen in [2, 3], in underwater, acoustic signals are used for range measurements because radio signals cannot propagate much under water.

In many studies regarding UWSN, the main puzzle of computing RSS has been resolved circuitously. According to Patwari [16], most of the studies have presumed that the RSS value can be converted to the distance but the complication of conversion has been ignored. In [6], the authors have proposed two methods for determining the distance of sensors in underwater using the transmission loss (TL), which can be acquired from the RSS. They proved that, the method using Lambert-W function gives significantly better result than the Newton-Raphson method considering the possible environmental constraints. In addition, the simulations result strongly back their claim. The resultant value is also notably close to the actual value. The authors of [5] proposed a method for localization based on sensors anchored to the seabed and the mobile sensors try to communicate directly with these anchored nodes to determine their position. This scheme cannot be applied to dynamic environment.

Rahman [4] has introduced a method to localize underwater sensors using Cayley-Menger determinant. They have used bouncing technique to calculate the necessary distances between the beacon and the sensors. Moreover, they used only a single beacon to localize the sensors. The sensors are considered static and the beacon takes measurements from at least six randomly different positions. However, their proposed model gives significantly accurate results.

The authors of [14] proposed a method to calculate the coordinates of submerged static sensors using a single beacon. They used trilateration to solve the problem and they dealt with multipath fading during distance measurement. In [7], authors solved the equations of multilateral operation. They tried to determine the unknown position using nonlinear square optimization. However, as per [9], in a nonlinear equation system, it does not give surety of a unique solution. For example, in trilateration method, distance is the only data to measure the distance between the nodes. 
After analyzing the studies discussed above, we propose a new mechanism to find out the coordinates of mobile submerged sensors, using a single beacon at the water surface. In addition, as in [8], to obtain primary subsets of nodes the precise conditions were vindicated using rigidity principle.

This paper is arranged as follows: solvable configuration and problem domain are described in Section II. Section III explains the technique for distance calculations. In Section IV, the theoretical method to determine the static sensors coordinates explained. In Section $\mathrm{V}$, mechanisms for determining the mobile sensors coordination is explained. In Section VI, analysis part is explained. Section VII discusses simulation results and at last, conclusions and future possible works are explained in Section VIII.

\section{Proposed CONFIGURATION}

\section{A. Problem Domain}

In the proposed method at least 3 sensor node and one beacon node is necessary to determine the coordinates of the mobile submerged sensors. The beacon is floating at the water surface. The distance between the sensors and the beacon are measured using a method based on Lambert function, as described in Section III. Usually a buoy or boat is used as a beacon and the sensors are deployed underwater in aquatic environment such as ocean or river. All the sensors are supposed to be in the same plane in underwater, which is parallel to the plane of the water surface where the beacon is, shown in Fig. 1 and 2.

We assume for simplicity, the sensors are Autonomous Underwater Vehicle (AUV), having static speed; and all sensors are moving in same direction. For six different positions of the sensors, same numbers of random different positions of the beacon are needed to take the measurements of the distance in between the sensors and the beacons. In the proposed model, the sensors generate acoustic signals in a predefined frequency. Then the beacon calculates transmission loss from RSS and calculates the distance to sensors. A solvable configuration of three sensors with the beacon is shown in Fig. 2. As stated by [11], the proposed model works in underwater within 1.8-323m depth. Moreover, as specified in [13], for acoustic signals, the method works for a frequency range below $50 \mathrm{kHz}$.

Our proposed model has a wide range of practical applications as most of the research and explorations of ocean take place in shallow water.

\section{B. Environmental Constraints}

The environment of underwater is more hostile than terrestrial environment. There are many environmental variables such as corrosion by salt water, the node's movements by the ocean current, attenuation distortions, issues of multi-path and difficulty of sensor nodes' deployment. In [13] we see that, it is quite complex and difficult to process and gather the information of the environment through ocean data communication due to the constraints of underwater environment unlike the terrestrial environment.
Acoustic signal is slower but propagates much further comparing to the radio signal. Again, the transmission loss is affected by temperature, depth, salinity, scattering, diffraction etc. As in [15], how these previously mentioned factors affect the transmission loss is not considered in this study and transmission loss is taken as a variable TL.

\section{Distance Determination for CAyley Menger USING LAMBERT-W FUNCTION}

Assumptions:

- The sensors can generate acoustic signals with a predefined frequency.

- While measuring distances, the factors that affect transmission loss is considered.

- Base for all the sensors is same and the base is of tetrahedron shape.

- All sensor nodes will have a fixed ID.

\section{A. Underwater Acoustic Transmission Loss Calculation}

There are two types of acoustic sound loss in underwater. These are classified as attenuation loss and spreading loss. Spreading loss includes spherical and cylindrical loss. In addition, attenuation loss includes absorption, leakage from ducts, scattering and diffraction. For simplicity, we only consider the transmission medium losses. For a distance D,

$\mathrm{TL}_{\mathrm{sph}}=20 \log (\mathrm{D})$, Spherical

$\mathrm{TL}_{\mathrm{cyl}}=10 \log (\mathrm{D})$, Cylindrical

So, total transmission loss we get from (1) and (2) is,

$\mathrm{TL}_{\text {total }}=\mathrm{TL}_{\mathrm{sph}}+\mathrm{TL}_{\text {cyl }}+10^{-3} \alpha \mathrm{D}$

Here, $\alpha$ is the absorption co-efficient, as per the Thorp absorption coefficient model.

$\alpha=1.0936\left[\frac{0.1 f 2}{1+f 2}+\frac{40 f 2}{4100+f 2}\right]$

Here, 1.0936 is multiplied to change the unit it to $\mathrm{dBkm}^{-1}$. As stated by [11], under a wide variety of condition, spherical data fits the measured data. So, by reducing (3) and (1),

$\mathrm{TL}=\frac{20 \ln (D)}{\ln (10)}+\frac{\alpha D}{1000}$

We will need to convert (5) into Lambert function to find a solution for the distance $D$.

Here, the Lambert-W function is

$\mathrm{Y}=\mathrm{AX} \mathrm{e}^{\mathrm{AX}}=\mathrm{W}(\mathrm{X})$

We need to find Lambert function $X=W(Y)$. Now, considering $X=D$ from (6), we will have,

$\mathrm{Y}=\mathrm{AX} \mathrm{e}^{\mathrm{AX}}$

$\frac{Y}{A}=\mathrm{D} \cdot \mathrm{e}^{\mathrm{A} \cdot \mathrm{D}}$

$\ln \left(\frac{Y}{A}\right)=\ln (\mathrm{D})+\mathrm{A} \cdot \mathrm{D}$ 
Let's consider, $\gamma=\ln (10) / 20$, then,

$\frac{\ln (Y / A)}{\gamma}=\frac{\ln (D)+A \cdot D}{\gamma}$

To derive (5), we must have these two conditions,

$\left(\frac{A}{\gamma}\right)=\left(\frac{\alpha}{1000}\right)$ and

$\frac{\ln (Y / A)}{\gamma}=\mathrm{TL}$

By solving them we get,

$A=(\gamma \alpha / 1000)$,

$\mathrm{Y}=\mathrm{Ae}^{\gamma \mathrm{TL}}$

\section{B. Distance Measurements using Lambert-W Function}

The Lambert-W function, is the multi valued inverse of $\omega \rightarrow \omega e^{\omega}$ defined by,

$z=(z)^{\mathrm{W}(\mathrm{z})}$

Where, $z$ and $W(z)$ can be complex. The sub-domain of both real and positive is used.

Here, $z$ is the transmission loss (TL). There is exactly one $\omega \geq 0$ for each $z \geq 0$, so $W$ returns a single value as distance.

Now,

$\omega_{1}=p-1$, where $p=\sqrt{2(e Y+1)}$

Using Halley Method, iterating toward $W(Y)$ from (12),

$\omega_{j}+1=\omega_{j}-\frac{\omega_{j} \mathrm{e}^{\omega_{j}-z}}{\mathrm{e} \omega_{j}\left(\omega_{j}+1\right)-\left(\left(\omega_{j}+2\right)\left(\omega_{j} \mathrm{e}^{\left.\left.\omega_{j}-z\right) /\left(2 \omega_{j}+2\right)\right)}\right.\right.}$

This solves (11) for $\omega$ where $z>0$. Accordingly,

$\mathrm{Y}=\mathrm{AXe}^{\mathrm{AX}} \quad \therefore \mathrm{X}=\frac{W(Y)}{A}$

From (14) and (10), we can write the final equation of Distance $(D)$ via Lambert function,

$\mathrm{D}=\frac{20000 \times W\left((\ln (10) / 20000) \alpha e^{T L}\right)}{\alpha \ln (10)}$

\section{CoORdinates Determination of Static Sensors USING CAYLEY MENGER}

\section{A. Determining Coordinates of the Sensor Nodes}

The goal of localization of the sensor nodes is to determine the precise position of the sensors. The only measurement here is to measure the distance. However, in nonlinear system, the degree of freedom analysis does not guarantee a singular solution. Multilateration or trilateration techniques are some nonlinear system, which are used to localize the sensors in some or full. According to Guevara [10], the convergence of Bayesian methods and optimization algorithms heavily depends on primary conditions used. They linearize the trilateration equations to overcome convergence problem.

In Fig. 1, the initial position of the beacon and the sensors are shown. The position of the beacon is $S_{j},(j=4,5 \ldots 9)$ and three sensor nodes are $\mathrm{S}_{\mathrm{i}},(\mathrm{i}=1,2,3)$. Without affecting generality, a coordinate system can be defined with respect to one of the sensor $S_{i}(i=1,2,3)$ as the origin $(0,0,0)$ of the system. Now the trilateration equation can be formed. The distance between beacon and the sensors are weighed data. Again, inter node distances $\mathrm{d}_{12}, \mathrm{~d}_{13}, \mathrm{~d}_{23}$ and volume of the tetrahedron $\mathrm{V}_{\mathrm{t}}$, are unknown. We write the equations based on the local positioning system configuration of Fig. 1. For that using Cayley-Menger determinant, the volume of tetrahedron $\mathrm{V}_{\mathrm{t}}$ is expressed as followings:

$288 V_{t}^{2}=\left|\begin{array}{ccccc}0 & 1 & 1 & 1 & 1 \\ 1 & 0 & d^{2}{ }_{12} & d^{2}{ }_{13} & d^{2}{ }_{14} \\ 1 & d^{2}{ }_{12} & 0 & d^{2}{ }_{23} & d^{2}{ }_{24} \\ 1 & d^{2}{ }_{13} & d^{2}{ }_{23} & 0 & d^{2}{ }_{34} \\ 1 & d^{2}{ }_{14} & d^{2}{ }_{24} & d^{2}{ }_{34} & 0\end{array}\right|$

$\mathrm{d}^{2}{ }_{34}\left(\mathrm{~d}^{2}{ }_{12}-\mathrm{d}^{2}{ }_{23}-\mathrm{d}_{13}^{2}\right)+\mathrm{d}^{2}{ }_{14}\left(\frac{\mathrm{d}^{4}{ }_{23}}{\mathrm{~d}^{2}{ }_{12}}-\mathrm{d}^{2}{ }_{23}-\frac{\mathrm{d}^{2}{ }_{13} \mathrm{~d}^{2}{ }_{23}}{\mathrm{~d}^{2}{ }_{12}}\right)+$

$\mathrm{d}^{2}{ }_{24}\left(\frac{\mathrm{d}^{4}{ }_{13}}{\mathrm{~d}^{2}{ }_{12}}-\frac{\mathrm{d}^{2}{ }_{13} \mathrm{~d}^{2}{ }_{23}}{\mathrm{~d}^{2}{ }_{12}}-\mathrm{d}^{2}{ }_{13}\right)-\left(\mathrm{d}^{2}{ }_{14} \mathrm{~d}^{2}{ }_{24}+\mathrm{d}^{2}{ }_{14} \mathrm{~d}^{2}{ }_{34}-\mathrm{d}^{2}{ }_{24} \mathrm{~d}^{2}{ }_{34}-\right.$

$\left.\mathrm{d}^{4}{ }_{14}\right) \frac{\mathrm{d}^{2}{ }_{23}}{\mathrm{~d}^{2}{ }_{12}}-\left(\mathrm{d}^{2}{ }_{34} \mathrm{~d}^{2}{ }_{24}-\mathrm{d}^{2}{ }_{14} \mathrm{~d}^{2}{ }_{34}+\mathrm{d}^{2}{ }_{14} \mathrm{~d}^{2}{ }_{24}-\mathrm{d}^{4}{ }_{24}\right) \frac{\mathrm{d}^{2}{ }_{13}}{\mathrm{~d}^{2}{ }_{12}}+$

$\left(\frac{144 \mathrm{v}_{\mathrm{t}}{ }^{2}}{\mathrm{~d}^{2}{ }_{12}}+\mathrm{d}_{13}{ }^{2} \mathrm{~d}_{23}{ }^{2}\right)=\left(\mathrm{d}^{2}{ }_{24} \mathrm{~d}^{2}{ }_{34}-\mathrm{d}_{34}^{4}+\mathrm{d}^{2}{ }_{14} \mathrm{~d}^{2}{ }_{34}-\mathrm{d}^{2}{ }_{14} \mathrm{~d}^{2}{ }_{24}\right)$

Here the unknown terms are,

$\left(d^{2}{ }_{12}-d^{2}{ }_{23}-d^{2}{ }_{13}\right),\left(\frac{d^{4}{ }_{13}}{d^{2}{ }_{12}}-\frac{d^{2}{ }_{13} d^{2}{ }_{23}}{d^{2}{ }_{12}}-d^{2}{ }_{13}\right), \frac{d^{2}{ }_{23}}{d^{2}{ }_{12}}, \frac{d^{2}{ }_{13}}{d^{2}{ }_{12}}$,

$\left(\frac{144 \mathrm{v}_{\mathrm{t}}{ }^{2}}{\mathrm{~d}^{2}{ }_{12}}+\mathrm{d}_{13}{ }^{2} \mathrm{~d}_{23}{ }^{2}\right)$ and $\left(\frac{\mathrm{d}^{4}{ }_{23}}{\mathrm{~d}^{4}{ }_{12}}-\mathrm{d}^{2}{ }_{23}-\frac{\mathrm{d}^{2}{ }_{13} \mathrm{~d}^{2}{ }_{23}}{\mathrm{~d}^{2}{ }_{12}}\right)$

By grouping and expanding known-unknown variables, we get,

$\mathrm{d}^{2}{ }_{14} \mathrm{X}_{1}+\mathrm{d}^{2}{ }_{24} \mathrm{X}_{2}+\mathrm{d}^{2}{ }_{34} \mathrm{X}_{3}-\left(\mathrm{d}^{2}{ }_{14}-\mathrm{d}^{2}{ }_{34}\right)\left(\mathrm{d}_{24}^{2}-\mathrm{d}^{2}{ }_{14}\right) \mathrm{X}_{4}-\left(\mathrm{d}^{2}{ }_{24}-\right.$

$\left.\mathrm{d}^{2}{ }_{14}\right)\left(\mathrm{d}^{2}{ }_{34}-\mathrm{d}^{2}{ }_{24}\right) \mathrm{X}_{5}+\mathrm{X}_{6}=\left(\mathrm{d}^{2}{ }_{24}-\mathrm{d}^{2}{ }_{34}\right)\left(\mathrm{d}_{34}^{2}-\mathrm{d}^{2}{ }_{14}\right)$

Equation (17) becomes as the linear shape of $\mathrm{a}_{1} \mathrm{x}_{1}+\mathrm{a}_{2} \mathrm{x}_{2}+$ $a_{3} x_{3}+\cdots \cdots+a_{n} x_{n}=b_{1}$. We need at least six measurements as we have six unknowns in (17). And this can be performed by following the same approach described in section earlier, moving the beacon $S_{j},(j=4,5 \ldots 9)$ to six different positions and measuring the distances in the vicinity of $\mathrm{P}_{4}$. Finally, we get $m$ number of linear equations of the form,

$a_{11} x_{1}+a_{12} x_{2}+\ldots \ldots+a_{1 n} x_{n}=b_{1}$
$a_{21} x_{1}+a_{22} x_{2}+\ldots \ldots+a_{2 n} x_{n}=b_{2}$
$a_{m 1} x_{1}+a_{m 2} x_{2}+\ldots \ldots+a_{m n} x_{n}=b_{m}$

By omitting references to the variables, the system of (18) can be represented by the augmented matrix of the system. Here, the first linear equation is represented by the first row of the array and so on. We can express it in a linear form, which is $A X=b$. Then the equations can be written as:

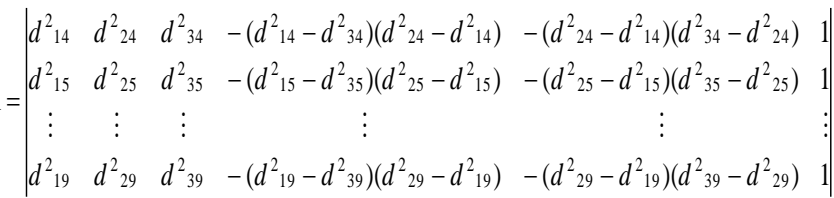




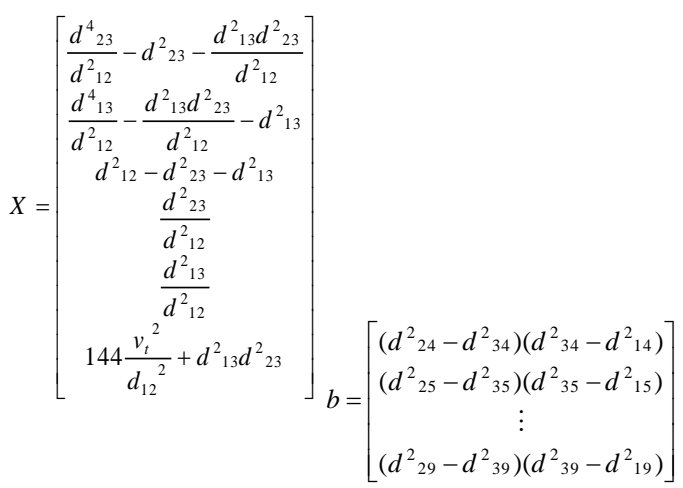

After finding the values of $\mathrm{X}\left(\mathrm{X}_{1}, \mathrm{X}_{2}, \mathrm{X}_{3}, \mathrm{X}_{4}, \mathrm{X}_{5}, \mathrm{X}_{6}\right)$ we calculate $\mathrm{d}_{12}, \mathrm{~d}_{23}, \mathrm{~d}_{13}$ as follows:

$\mathrm{d}^{2}{ }_{12}=\frac{\mathrm{X} 4}{1-\mathrm{X} 4-\mathrm{X} 5}, \quad \mathrm{~d}^{2}{ }_{13}=\frac{\mathrm{X} 3 \mathrm{X}_{5}}{1-\mathrm{X} 4-\mathrm{X} 5}, \quad \mathrm{~d}^{2}{ }_{23}=\frac{\mathrm{X} 3 \mathrm{X}_{4}}{1-\mathrm{X} 4-\mathrm{X} 5}$

As we assume that the submerged sensors coordinate are $S_{1}=(0,0,0), S_{2}=\left(0, y_{2}, 0\right)$ and $S_{3}=\left(x_{3}, y_{3}, 0\right)$ then with respect to coordinates of the sensors the inter sensor distances could be written as follows:

$\mathrm{d}^{2}{ }_{12}=\mathrm{y}_{2}{ }^{2}, \quad \mathrm{~d}^{2}{ }_{13}=\mathrm{x}_{3}{ }^{2}+\mathrm{y}_{3}{ }^{2}, \quad \mathrm{~d}^{2}{ }_{23}=\mathrm{x}_{3}{ }^{2}+\left(\mathrm{y}_{3}{ }^{2}-\mathrm{y}_{2}{ }^{2}\right)$

After finding the values above, we can calculate the unknown values as follows [4]:

$\mathrm{y}_{2}=\mathrm{d}_{12}$,

$\mathrm{y}_{3}=\frac{\mathrm{d}^{2}{ }_{12}+\mathrm{d}^{2}{ }_{13}-\mathrm{d}^{2}{ }_{23}}{2 \mathrm{~d}_{12}}$

$x_{3}=\sqrt{\left(d^{2}{ }_{13}-\left(\frac{d^{2}{ }_{12}+d^{2} 13-d^{2} 23}{2 d_{12}}\right)^{2}\right)}$

Here, $\mathrm{d}_{12}, \mathrm{~d}_{13}, \mathrm{~d}_{23}$ are computed distance. The sensors coordinate with respect to $S_{1}$ are given in Table I.

\section{B. Determining the Coordinates of the Sensor Nodes}

Now, the position of the beacon has to be in origin $(0,0,0)$ to determine the sensors coordinates. As we can calculate other sensors coordinates with respect to $S_{1}$, we only need to find the coordinate of sensor $S_{1}$ with respect to the beacon. Now the coordinates of sensor $S_{1}$ with respect to the beacon node can be determined by following these steps.

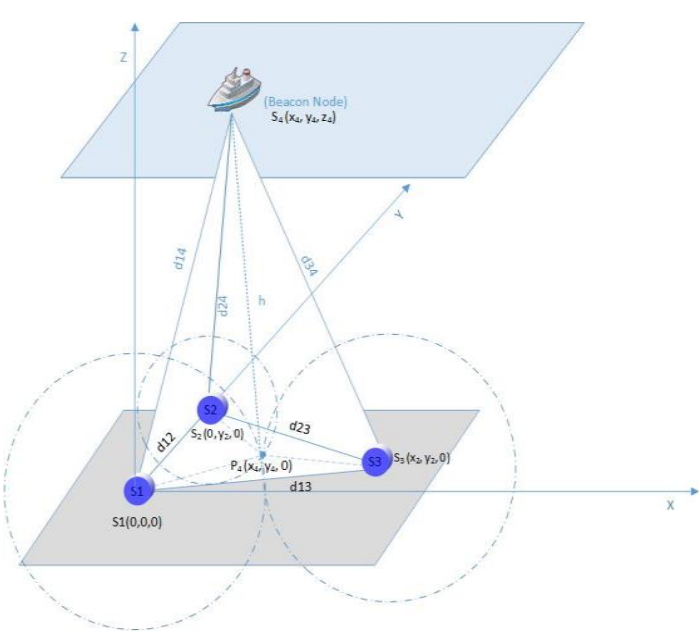

Fig. 1. Coordinate Determinations with Single Beacon.
TABLE. I. COORDINATES OF THE SENSORS WITH RESPECT TO $\mathrm{S}_{1}$

\begin{tabular}{|l|l|}
\hline Node & Coordinates \\
\hline $\mathrm{S}_{1}$ & $(0,0,0)$ \\
\hline $\mathrm{S}_{2}$ & $\left(0, \mathrm{~d}_{12}, 0\right)$ \\
\hline $\mathrm{S}_{3}$ & $\left.\left(\sqrt{\left(\mathrm{d}^{2}{ }_{13}-\left(\frac{\mathrm{d}^{2}{ }_{12}+\mathrm{d}^{2}{ }_{13}-\mathrm{d}_{23}{ }_{23}}{2 \mathrm{~d}_{12}}\right)\right.}\right),\left(\frac{\mathrm{d}^{2}{ }_{12}+\mathrm{d}^{2}{ }_{13}-\mathrm{d}^{2}{ }_{23}}{2 \mathrm{~d}_{12}}\right), 0\right)$ \\
\hline
\end{tabular}

According to [12], the vertical distance $h$ can be measured. After measuring $h$, we assume the projected coordinate of $\mathrm{S}_{4}$ is $\mathrm{P}_{4}\left(\mathrm{x}_{4}, \mathrm{y}_{4}, 0\right)$ on the plane $\mathrm{XY}$. To find $\mathrm{x}_{4}$ and $\mathrm{y}_{4}$, trilateration technique is applied, assuming the distances between sensors $\mathrm{S}_{1}, \mathrm{~S}_{2}, \mathrm{~S}_{3}$ and projected point $\mathrm{P}_{4}$ are $\mathrm{D}_{14}, \mathrm{D}_{24}$ and $\mathrm{D}_{34}$.

$\mathrm{D}^{2}{ }_{14}=\mathrm{x}_{4}{ }^{2}+\mathrm{y}_{4}^{2}$

$\mathrm{D}_{24}^{2}=\mathrm{x}_{4}^{2}+\left(\mathrm{y}_{4}-\mathrm{y}_{2}\right)^{2}$

$\mathrm{D}_{34}^{2}=\left(\mathrm{x}_{4}-\mathrm{x}_{3}\right)^{2}+\left(\mathrm{y}_{4}-\mathrm{y}_{3}\right)^{2}$

From (19), (20) and (21) we get the coordinates of projected beacon as follows $\mathrm{P}_{4}\left(\mathrm{x}_{4}, \mathrm{y}_{4}, \mathrm{z}_{4}\right)$.

$X_{4}=\sqrt{\frac{1}{2 D}\left(2 d_{12} D^{2}{ }_{14}-D^{2}{ }_{14}+D^{2}{ }_{24}+d^{2}{ }_{12}\right)}$,

$\mathrm{Y}_{4}=\frac{1}{2 \mathrm{~d}_{12}}\left(\mathrm{D}^{2}{ }_{14}-\mathrm{D}_{24}^{2}+\mathrm{d}^{2}{ }_{12}\right)$

As the hypotenuse of $\triangle \mathrm{S}_{1} \mathrm{P}_{4} \mathrm{~S}_{4}, \triangle \mathrm{S}_{2} \mathrm{P}_{4} \mathrm{~S}_{4}$ and $\triangle \mathrm{S}_{3} \mathrm{P}_{4} \mathrm{~S}_{4}$ are $\mathrm{d}_{14}, \mathrm{~d}_{24}$ and $\mathrm{d}_{34}$ respectively, so the distance $\mathrm{D}_{14}, \mathrm{D}_{24}$ and $\mathrm{D}_{34}$ is possible to obtain by implementing Pythagorean Theorem. Now, the coordinate of the beacon $\mathrm{S}_{4}\left(\mathrm{x}_{4}, \mathrm{y}_{4}, \mathrm{z}_{4}\right)$ will transform as $\left(\mathrm{x}_{4}, \mathrm{y}_{4}, \mathrm{~h}\right)$ where all elements are known.

$\mathrm{S}_{4}\left(\mathrm{x}_{4}, \mathrm{y}_{4}, 0\right)=S_{4}\left(\begin{array}{c}\left.\sqrt{\frac{1}{2 \mathrm{D}}\left(2 \mathrm{~d}_{12} \mathrm{D}^{2}{ }_{14}-\mathrm{D}^{2}{ }_{14}+\mathrm{D}^{2}{ }_{24}+\mathrm{d}^{2}{ }_{12}\right)}\right) \\ \frac{1}{2 \mathrm{~d}_{12}}\left(\mathrm{D}^{2}{ }_{14}-\mathrm{D}^{2}{ }_{24}+\mathrm{d}^{2}{ }_{12}\right), \mathrm{h}\end{array}\right)$

Applying linear transformation, the coordinate of the beacon node is replaced by the origin of the Cartesian system. The linear transformation would give the coordinates of other sensor nodes as in Table II.

TABLE. II. COORDINATES OF THE SENSORS WITH RESPECT TO $\mathrm{S}_{4}$

\begin{tabular}{|l|l|l|l|}
\hline Sensors & Coordinates & Sensors & Coordinates \\
\hline$S_{4}$ & $(0,0,0)$ & $S_{2}$ & $\left(-\mathrm{x}_{2}, \mathrm{y}_{2}-\mathrm{y}_{4},-\mathrm{z}_{4}\right)$ \\
\hline $\mathrm{S}_{1}$ & $\left(-\mathrm{x}_{4},-\mathrm{y}_{4},-\mathrm{z}_{4}\right)$ & $\mathrm{S}_{3}$ & $\left(-\mathrm{x}_{4}, \mathrm{y}_{2}-\mathrm{y}_{4},-\mathrm{z}_{4}\right)$ \\
\hline
\end{tabular}

\section{CoOrdinate Determination of Mobile SEnsors}

Initially, the distance between the sensors and the beacon are to be calculated with the help of Lambert-W function. Here, d11, d21 \& d31 are the distance between sensor $\mathrm{S}_{\mathrm{i}}{ }^{\prime} \mathrm{s}(\mathrm{i}=$ $1,2,3)$ initial position to beacons initial $\left(B_{k}=1\right)$ position, respectively. As both the beacon and sensors are mobile, the distance between beacons new position to sensors new position is to be calculated using the Lambert function, as mentioned in Section III. For $\mathrm{S}_{1}$, it is $\mathrm{d}_{2} 2$, as shown in Fig. 2. Concurrently, $\mathrm{d} 2{ }_{2} 2$ and $\mathrm{d} 3{ }_{2} 2$ is calculated for $S_{2}$ and $S_{3}$ following the same process. Here, in $\mathrm{dSi}_{j} \mathrm{~B}_{\mathrm{k}}, \mathrm{S}_{\mathrm{i}}(\mathrm{i}=1,2,3)$ is the sensor number, $\mathrm{j}(\mathrm{j}=1,2,3 \ldots 6)$ is the sensors position and $\mathrm{B}_{\mathrm{k}}(\mathrm{k}=1,2,3 \ldots 6)$ is the beacon's position. 


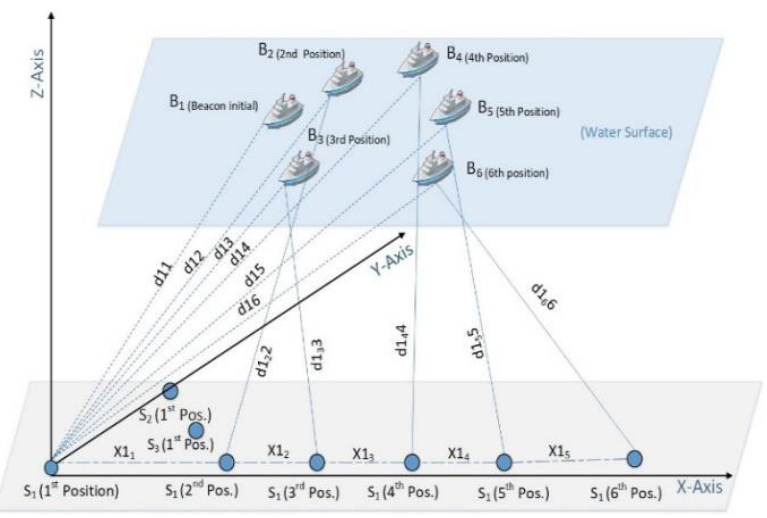

Fig. 2. Coordinate Determination of Mobile Sensors.

Now the distance from sensor's initial position to second position is calculated with the help of the sensors speed and the time beacon took to travel to its new position from previous position. Here, the sensors are moving in a stationary speed and fixed direction (x-axis).

$\mathrm{xSi}_{\mathrm{j}}=\mathrm{v}_{\mathrm{i}} \cdot \mathrm{t}_{\mathrm{m}}$; Here, $\mathrm{t}_{\mathrm{m}}=$ time between beacons $\mathrm{m}-1^{\text {th }}$ and $\mathrm{m}^{\text {th }}$ measurement and $v_{i}=$ sensor $S_{i}$ 's speed.

Now, applying Pythagorean Theorem the distance of the beacons new position to the sensors initial position is calculated.

$\mathrm{d}_{12}=\sqrt{\left(\mathrm{d} 1_{2} 2\right)^{2}+\left(\mathrm{x} 1_{1}\right)^{2}} ; \mathrm{d} 1_{2} 2=$ distance between sensor 1 's second position to beacons second position as in Fig. 2.

$\mathrm{d}_{22}=\sqrt{\left(\mathrm{d} 2_{2} 2\right)^{2}+\left(\mathrm{x} 2_{1}\right)^{2}} ; \mathrm{d} 2_{2} 2=$ distance between sensor 2 's second position to beacons second position.

$\mathrm{d}_{32}=\sqrt{\left(\mathrm{d} 3_{2} 2\right)^{2}+\left(\mathrm{x} 3_{1}\right)^{2}} ; \mathrm{d} 3_{2} 2=$ distance between sensor 3 's second position to beacons second position.

This process is repeated six times from six random positions of the beacon with six different positions of the sensors to find the distance from beacon's new position to sensors initial position. For sensor 1, the process is shown in Fig. 2.

Then calculating those distances, the values of augmented matrix is originated, as in Section IV. From that matrix six unknowns $\left(X_{n}, n=1,2,3 \ldots 6\right)$ of (16) is found. After that, the inter sensor distances of the initial position is generated, as alluded in Section IV. Thereafter, the coordinate of the Projected point $\mathrm{P}_{4}$ as shown in Fig. 1 and distances from sensors to $\mathrm{P}_{4}$ is calculated. Then the initial coordinate of the sensors is found as in Table I. In addition, after applying linear transformation with respect to beacon Table II is generated.

By, adding the distance travelled by the sensors from the first position to the sixth position with $\mathrm{x}$-axis; the current coordinates of the sensors are found, as shown in Table III.

$x i=x i_{1}+x i_{2}+x i_{3}+x i_{4}+x i_{5}$

TABLE. III. CURRENT COORDINATES

\begin{tabular}{|l|l|l|l|}
\hline Sensors & Coordinates & Sensors & Coordinates \\
\hline$S_{4}$ & $(0,0,0)$ & $S_{2}$ & $\left(-x_{2}+x 2, y_{2}-y_{4},-z_{4}\right)$ \\
\hline$S_{1}$ & $\left(-x_{4}+x_{1} 1,-y_{4},-z_{4}\right)$ & $S_{3}$ & $\left(-x_{4}+x 3, y_{2}-y_{4},-z_{4}\right)$ \\
\hline
\end{tabular}

\section{ANALYSIS}

Our method is for a specific scenario, where only one beacon is necessary to determine the coordinates of mobile submerged sensors. Most of the localization methods depend on distance measurements and usually lots of sensors and beacons are deployed. Therefore, precise measurement of the distance is one of the most important factors for accurate localization.

In our proposed model, the beacon floats on the water surface and a minimum of three mobile sensors are deployed underwater. Most importantly, our method determines the 3D coordinates of mobile sensors with respect to the beacon node. So the coordinates of the sensors are calculated more accurately as the coordinate of the beacon node can be measured precisely using Global Positioning System (GPS).

\section{A. Distance Measurement Complexity}

The limitations of underwater acoustic signal are considered in this model during distance measurements. The method is simple and understandable but it gives accurate results when the transmission loss of the signal is calculated precisely. Considering some of the practical applications, a pragmatic assumption is considered where the beacon should have the capability to receive signal $\left(\mathrm{R}_{\mathrm{x}}\right)$. On the other hand, the sensors would transmit signal $\left(\mathrm{T}_{\mathrm{x}}\right)$. In Fig. 3 we see the relation between the distance and transmission loss as the distance is higher, the rate of transmission loss is also high.

The transmission loss depends on several factors like salinity, depth, acidity, temperature, bubble curtain or other damping structure. While measuring TL, these factors must be under consideration. For a constant frequency, the distance increases with the increase in transmission loss and vice versa. Fig. 3 shows the relation between transmission loss and distance.

\section{B. Error Generation}

In our method, we have found less error while measuring the distance because the distance measurement method only depends on frequency and transmission loss. The sensors generate the signals initially instead of decoding a message from the RSS, as mentioned in Section III.

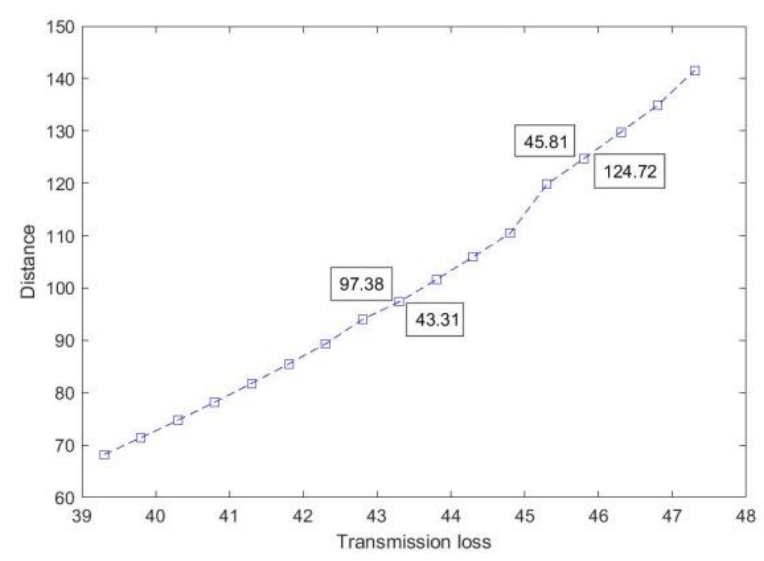

Fig. 3. Relation between the Distance and Transmission Loss. 
In acoustic signal propagation, the transmission loss depends on various factors. Therefore, more accurate transmission loss calculation would give a better distance measurement resulting initial and mobile coordinate estimation with less error. In our technique, we have not used any bouncing technique while measuring the distance as the bouncing technique suffers from multipath fading.

\section{SimUlation RESULTS AND DisCUSSION}

The proposed strategy is simulated using MATLAB to validate the mathematical model. The sensors are placed randomly at $(0,0,0),(0,70,0)$ and $(85,90,0)$. The beacon is randomly moved in a plane, parallel to the XY plane. The positions of the beacon are given in Table IV.

One of the sensors is situated at the origin and another one on the y-axis to avoid computational complexity. We have added some Gaussian Noise with the Euclidean distance to find the coordinates of the sensors. After implementing the trilateration, the final coordinates of the sensors are found. Moreover, by using the method based on Lambert function for distance measurement, at a static frequency of $45 \mathrm{kHz}$, the initial coordinates of the sensors are found. After that, by adding the distance moved by the sensors with respect to the $\mathrm{x}$-axis, the current coordinates are found. Here, the distance travelled by the sensors is $239.2395 \mathrm{~m}$.

In Fig. 4, Initial coordinates for sensor $S_{2}$ using Lambert function for distance measurements is denoted as S2 and current coordinate of sensor $\mathrm{S}_{2}$ is denoted as $\mathrm{S} 2$ '.

In Fig. 5, Coordinates of sensor $S_{1}, S_{2}$ and $S_{3}$ using Euclidean distances are denoted as $S_{1}, S_{2}, S_{3}$ and coordinates using the method established on Lambert function for distance measurements are denoted as $S_{1}{ }^{\prime}, S_{2}{ }^{\prime}, S_{3}{ }^{\prime}$, respectively.

TABLE. IV. BEACONS COORDINATES

\begin{tabular}{|l|l|l|l|l|l|l|}
\hline & $\mathbf{B}_{\mathbf{1}}$ & $\mathbf{B}_{\mathbf{2}}$ & $\mathbf{B}_{\mathbf{3}}$ & $\mathbf{B}_{\mathbf{4}}$ & $\mathbf{B}_{\mathbf{5}}$ & $\mathbf{B}_{\mathbf{6}}$ \\
\hline $\mathbf{x}$ & 100 & 90 & 80 & -10 & -20 & -30 \\
\hline $\mathbf{y}$ & 90 & 80 & 70 & 60 & -60 & -90 \\
\hline $\mathbf{z}$ & 70 & 70 & 70 & 70 & 70 & 70 \\
\hline
\end{tabular}

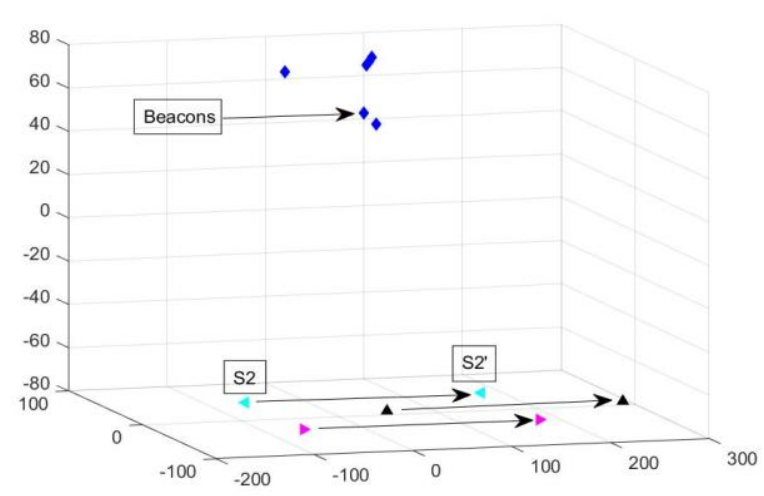

Fig. 4. Current Coordinates of the Sensors.

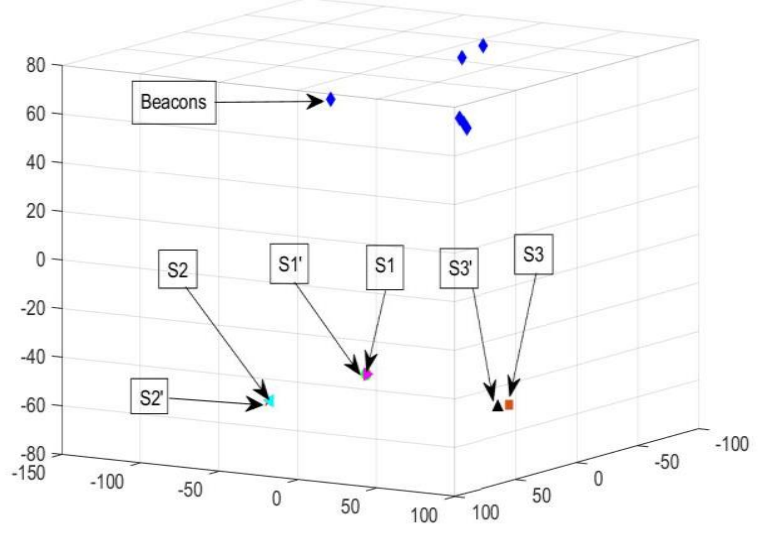

Fig. 5. Comparison between Final Coordinates using Euclidean Distances and Experimental Distances.

Table V compares the error in final coordinates of sensor $\mathrm{S}_{1}, \mathrm{~S}_{2}$ and $\mathrm{S}_{3}$ as the distances between the beacon and sensors are calculated using the proposed method with when distances between the sensors and beacon are calculated using Euclidean distances. Here, the coordinate of $S_{3}$ is showing maximum error. Error in $S_{1}$ and $S_{2}$ are negligible.

The positional errors of the sensors are given in Table VI. Error for $S_{1}$ is negligible where the error is less than a meter. In addition, for $S_{2}$ it is a bit above $1.5 \mathrm{~m}$. The error for $S_{3}$ is comparatively high as it is above $7 \mathrm{~m}$.

Positional error of sensors generated with the proposed model is moderate. This also proves the importance of precise evaluation of TL. Table VII compares the error in coordinates at different frequencies. The actual coordinate is measured at frequency $45 \mathrm{kHz}$, which is denoted at Table $\mathrm{V}$.

The percentage of error increases with the error in frequency.

TABLE. V. COORDINATE ERROR OF SENSORS

\begin{tabular}{|c|c|c|c|c|c|}
\hline \multirow{2}{*}{ Sensors } & \multirow{2}{*}{$\begin{array}{l}\text { Actual } \\
\text { Coordinate } \\
(\mathbf{x}, \mathbf{y}, \mathbf{z})\end{array}$} & \multirow{2}{*}{$\begin{array}{l}\text { Experimental } \\
\text { Coordinates (x, } \\
\mathbf{y}, \mathbf{z})\end{array}$} & \multicolumn{3}{|c|}{$\begin{array}{l}\text { Percentage of Error } \\
(\%)\end{array}$} \\
\hline & & & $x$ & $y$ & $z$ \\
\hline$S_{1}$ & $\begin{array}{l}(-102.87, \\
-88.44, \\
-70)\end{array}$ & $\begin{array}{l}(102.73, \\
-88.61, \\
-70)\end{array}$ & 0.16 & -0.19 & 0 \\
\hline $\mathrm{S}_{2}$ & $\begin{array}{l}(-102.88, \\
-8.09, \\
-70)\end{array}$ & $\begin{array}{l}(-102.73, \\
-9.78, \\
-70)\end{array}$ & 0.14 & -20.88 & 0 \\
\hline $\mathrm{S}_{3}$ & $\begin{array}{l}(13.97, \\
-55.19, \\
-70)\end{array}$ & $\begin{array}{l}(12.19,-8.12, \\
-70)\end{array}$ & 12.79 & 12.80 & 0 \\
\hline
\end{tabular}

TABLE. VI. POSITIIONAL ERROR OF SENSORS

\begin{tabular}{|l|l|l|}
\hline $\mathbf{S}_{\mathbf{1}}$ & $\mathbf{S}_{\mathbf{2}}$ & $\mathbf{S}_{\mathbf{3}}$ \\
\hline $0.2288 \mathrm{~m}$ & $1.694 \mathrm{~m}$ & $7.287 \mathrm{~m}$ \\
\hline
\end{tabular}


TABLE. VII. ERROR COMPARISION AT DIFFERENT FREQUENCIES

\begin{tabular}{|l|l|l|l|l|}
\hline Sensors & Frequency & $\mathbf{x}$ & $\mathbf{y}$ & $\mathbf{z}$ \\
\hline \multirow{3}{*}{$\mathrm{S}_{1}$} & $44.95 \mathrm{kHz}$ & $0.058 \%$ & $0.167 \%$ & $0 \%$ \\
\cline { 2 - 5 } & $44.85 \mathrm{kHz}$ & $0.174 \%$ & $0.501 \%$ & $0 \%$ \\
\cline { 2 - 5 } & $44.75 \mathrm{kHz}$ & $0.286 \%$ & $0.829 \%$ & $0 \%$ \\
\hline \multirow{3}{*}{$\mathrm{S}_{2}$} & $44.95 \mathrm{kHz}$ & $0.058 \%$ & $-10.041 \%$ & $0 \%$ \\
\cline { 2 - 5 } & $44.85 \mathrm{kHz}$ & $0.174 \%$ & $-27.828 \%$ & $0 \%$ \\
\cline { 2 - 5 } & $44.75 \mathrm{kHz}$ & $0.286 \%$ & $-43.122 \%$ & $0 \%$ \\
\hline \multirow{3}{*}{$\mathrm{S}_{3}$} & $44.95 \mathrm{kHz}$ & $12.705 \%$ & $12.303 \%$ & $0 \%$ \\
\cline { 2 - 5 } & $44.85 \mathrm{kHz}$ & $41.279 \%$ & $32.400 \%$ & $0 \%$ \\
\cline { 2 - 5 } & $44.75 \mathrm{kHz}$ & $71.086 \%$ & $48.042 \%$ & $0 \%$ \\
\hline
\end{tabular}

\section{CONCLUSION AND FUTURE WORK}

In this paper, a mathematical model is presented to localize submerged mobile sensors using only one beacon node. A method based on Lambert-W function is used to measure the distances between the beacon and the sensors and the coordinates of the sensors are determined using CayleyMenger determinant. Where all the sensors are moving in the same direction along the $\mathrm{x}$-axis, and the sensors speed are static and known. Moreover, our distance measurement technique contributes less error and does not need any kind of synchronization. Simulation result validates that there are some error between the Euclidian distance and the experimented distance; resulting in erroneous coordinates. However, precise measurement of Transmission Loss gives accurate distance; finally leading to flawless coordinate determination. Therefore, the accurate measurement of Transmission Loss gets utmost priority in this approach.

In future, we plan to localize the sensors, moving in different directions and unknown speed.

\section{REFERENCES}

[1]. H.P. Tan, R. Diamant, W.K.G. Seah, and M. Wanldmeyer, "A survey of techniques and challenges in underwater localization," Ocean Engineering, vol. 38, pp.1663-1676, 2011.

[2]. J.H. Cui, J. Kong, M. Gerla, and S. Zhou, "The challenges of building mobile underwater wireless networks for aquatic applications," Network, IEEE, vol. 20, pp. 12-18, 2006.
[3]. P. Xie, J. H. Cui, and L. Lao, "VBF: vector-based forwarding protocol for underwater sensor networks," Networking Technologies, Services, and Protocols; Performance of Computer and Communication Networks; Mobile and Wireless Communications Systems, pp. 1216-1221, 2006.

[4]. A. Rahman, V. Muthukkumarasamy, E. Sithirasenan, "Coordinates determination of submerged sensors using cayley-menger determinant," IEEE International Conference on Distributed Computing in Sensor System, 2013.

[5]. T.C. Austin, R. P. Stokey, and K. M. Sharp, "PARADIGM: a buoybased system for AUV navigation and tracking," in OCEANS 2000 MTS/IEEE Conference and Exhibition, 2000, pp. 935-938 vol.2.

[6]. M. Hosseini, H. Chizari, T. Poston, M. Bt. Salleh, A. H. Abdullah, "Efficient underwater RSS Value to distance Inversion Using Lambert Function," Mathematical Problems in Engineering, vol.2014, Article ID 175275, 2014.

[7]. P. Duff and H. Muller, "Auto calibration algorithm for ultrasonic location systems," in Wearable Computers, 2003. Proceedings. Seventh IEEE International Symposium on, 2003, pp.62-68.

[8]. E. Olson, J. Leonard, and S. Teller, "Robust range-only beacon localization," in Autonomous Underwater Vehicles, 2004 IEEE/OES, 2004, pp.66-75.

[9]. J. Guevara, A. R. Jimenez, A. S. Morse, J. Fang, J. C. Prieto, and F. Seco, "Auto-localization in Local Positioning Systems: A closedform range-only solution," in Industrial Electronics (ISIE), 2010 IEEE International Symposium on, 2010, pp.2834-2840.

[10]. J. Guevara, A. Jiménez, J. Prieto, and F. Seco, "Auto-localization algorithm for local positioning systems," Ad Hoc Networks, 2012.

[11]. "Principles of Underwater Sound," Publishing, $3^{\text {rd }}$ edition, 1983.

[12]. R. M. Corless, G. H. Gonnet, D. E. G. Hare, D. J. Jeffrey, and D. E. Knuth, "On the Lambert-W function", Advances in Computational Mathematics, vol.5, no. 4, pp.329-359, 1996.

[13]. Yun, Nam-Yeol\&NamGung, Jung-Il \& Park, Hyun-Moon \& Park, Su-Hyeon\& Kim, Chang-Hwa, "The underwater environment monitoring system based on ocean oriented WSN (Wireless Sensor Network)," Journal of Korea Multimedia Society, 2010.

[14]. A. Rahman, V. Muthukkumarasamy, E. Sithirasenan, "Localization of submerged sensors using radio and acoustic signals with single beacon," Cichoń J., Gębala M., Klonowski M. (eds) Ad-hoc, Mobile, and Wireless Network, ADHOC-NOW, 2013.

[15]. M. Hosseini, H. Chizari, C. K. Soon, R. Budiarto "RSS-based distance measurement in underwater acoustic sensor networks: An application of the Lambert-W function", International Conference on Signal Processing and Communication System, 2010.

[16]. N. Patwari, P. Agrawal, "Calibration and Measurement of Signal Strength for Sensor Localization, IGI Global, 2009. 